\title{
Electron magnetic resonance study of multiwalled carbon nanotubes and carbon nanohorns
}

\author{
Ireneusz Stefaniuk ${ }^{1 *}$, Bogumil Cieniek ${ }^{1}$, and Iwona Rogalska $^{1}$ \\ ${ }^{1}$ Faculty of Mathematics and Natural Sciences, University of Rzeszow, S. Pigonia 1, 35-310 Rzeszow, Poland
}

\begin{abstract}
Temperature Electron Magnetic Resonance (EMR) measurements of multiwalled carbon nanotubes $(\mathrm{CNT})$ and carbon nanohorns $(\mathrm{CNH})$ were performed in the temperature range $5.2-300 \mathrm{~K}$. The asymmetric resonance lines with Dyson shape were observed. The g-value of multiwalled carbon nanotubes $(\mathrm{CNT})$ and carbon nanohorns $(\mathrm{CNH})$ was calculated. Analysis of the temperature dependences of the integral intensity of EMR spectra was carried out using the Curie-Weiss law and Curie temperature $\theta_{(\mathrm{CNT})}=$ $46,6 \mathrm{~K}$ and $\theta_{(\mathrm{CNT})}=8.6 \mathrm{~K}$ were obtained. We showed that the localization processes observed in nanocarbon materials lead to local quantum transport of spins or carriers.
\end{abstract}

\section{Introduction}

Since their discovery by Iijima in 1991 [1] carbon nanotubes have attracted increasing attention from the material science community due to their exceptional electronic and mechanical properties [2].

Carbon nanotubes are promising candidates for a variety of applications due to their uncommon and versatile electrical and mechanical properties which recently generated an active area of research [3]. Possible technological applications include, among others, nanotransistors $[4,5]$, spin-polarized electron sources [6], hydrogen storage [7, 8], flat display panels [9] and magnetic inks.

Single nanotubes have been used to fabricate transistors [10] and show potential as the building block for new nanotechnologies. Potential applications of nanotubes include using them as an electron source in field emission displays [11], as an optical limiting material [12] and as an electromagnetic interference shielding material. However, there remains a need to characterize the purity of carbon nanotube powders and to develop a mechanism to isolate nanotubes.

Quasi-graphitic carbon nanoparticles (CNs) were found to show very interesting behaviour with respect to the localization of charge carriers. Due to their large specific surface area, CNs are very sensitive to the adsorption of various molecules. Their electronic properties strongly depend on their structure and interactions between the molecules adsorbed in pores and the pore walls (host-guest interactions).

The magnetism of carbon-based materials (i.e. graphene, graphite, carbon nanotubes, and fullerenes) has recently been the subject of intense research [13-16]. These materials exhibit exotic magnetism such as ferromagnetism above room temperature, which is believed to be induced by defects in the graphitic network.

Magnetic and electronic properties of multiwall carbon nanotubes has been presented in [17-19].

The main aim of this work it characterization properties of multiwalled carbon nanotubes (CNT) and carbon nanohorns $(\mathrm{CNH})$ using Electron Magnetic Resonance (EMR). Serially produced material of CNT and $\mathrm{CNH}$ by Sigma-Aldrich was used as samples. Although measurements of similar nanoparticles are available in literature, but our target in future is to modify CNT and CNH to use it for example as sensors. This is the reason to measure EMR in wide temperature range from room down to liquid helium temperature.

\section{Experiment}

Multi walled carbon nanotubes (MWNTs, CNTs) were prepared by chemical vapor deposition (CVD). In chemical vapor deposition (CVD), a volatile precursor undergoes thermal decomposition at elevated temperatures to form a solid deposit on a substrate. The diameter of the tube ranges between $6-13 \mathrm{~nm}$ and the average length is $10 \mu \mathrm{m}$. The multiwalled nanotubes are stable in inert atmosphere up to a temperature of $3697^{\circ} \mathrm{C}$. CVD production method is followed by $\mathrm{HCl}$ demineralization. Single-walled carbon nanohorns are made of graphene sheets with long cone-shaped tips with the diameters of $2-5 \mathrm{~nm}$ and the length of $40-50 \mathrm{~nm}$. Thousands of carbon nanohorns form spherical aggregates of about $100 \mathrm{~nm}$ in diameter.

CNT multiwalled, more than $98 \%$ of carbon basis, and $\mathrm{CNH}$ as grown material was manufactured by Sigma-Aldrich.

The EMR spectra were investigated in a wide range of temperatures from $5 \mathrm{~K}$ up to $300 \mathrm{~K}$ using an EPR X-

* Corresponding author: istef@ur.edu.pl 
band spectrometer (Bruker multifrequency and multiresonance FT-EPR ELEXSYS E580). Measurements at low temperatures were carried out using an Oxford Instruments continuous-flow helium cryostat $(3.8 \mathrm{~K}$ to $400 \mathrm{~K}$ with the Helium Temperature Control System ER4112HV).

CNT and CNH samples were transferred into thinwalled capillary closed after filling. Samples have been exposed to atmospheric oxygen only during filling the capillary.

The EMR spectra for the samples described above are shown in Fig. 1. The shape of the EMR spectra is of a Dyson type [20], with a asymmetry parameter of the resonance line $\mathrm{a} / \mathrm{b}=1.5$ at $50 \mathrm{~K}$, where $\mathrm{a}$ and $\mathrm{b}$ are amplitudes of the low field and high field halves of the spectrum, respectively.

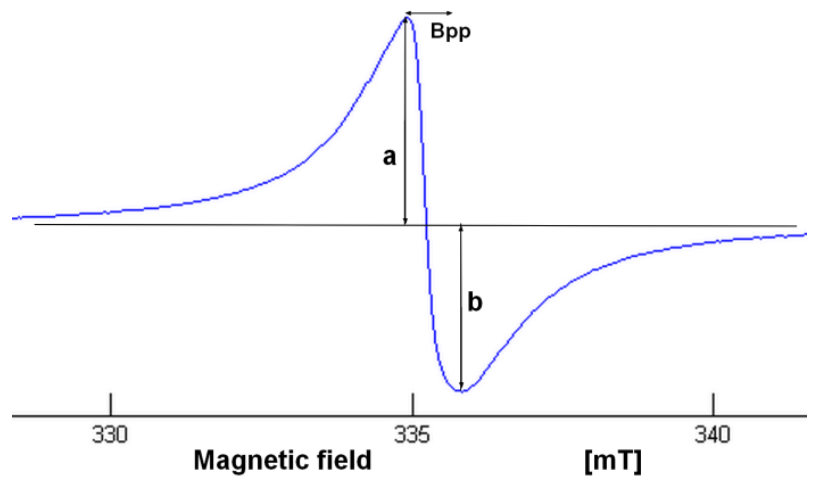

Fig. 1. Dysonian EMR signal of the $\mathrm{CNH}$. The characteristic points and parameters are shown.

In Fig. 2 EMR spectra of $\mathrm{CNT}$ and $\mathrm{CNH}$ acquired at temperature $50 \mathrm{~K}$ are shown. In Fig. 3 and 4 the temperature dependences of EMR spectra are shown.

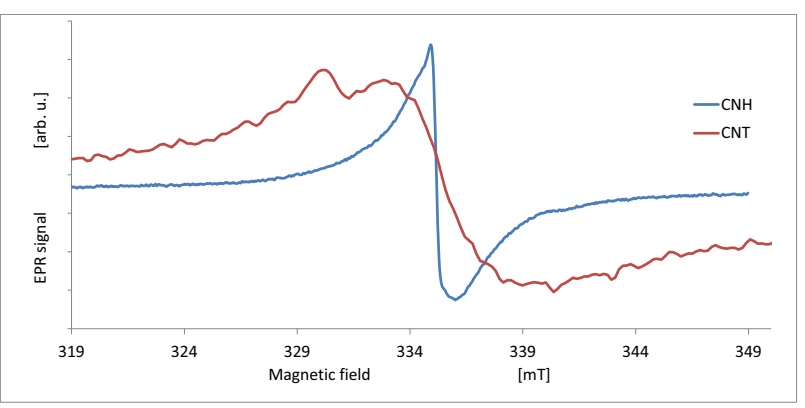

Fig. 2. The EMR spectra of the $\mathrm{CNT}$ and $\mathrm{CNH}$ at temperature $50 \mathrm{~K}$.

Two EMR signals (one broad and one narrow) were recorded at low temperatures for CNT sample. All spectra were asymmetric and could be well fitted to Dysonian shape. From these fits, the $g$ value (representing position), Bpp (peak-to-peak linewidth) and integral intensity were calculated.

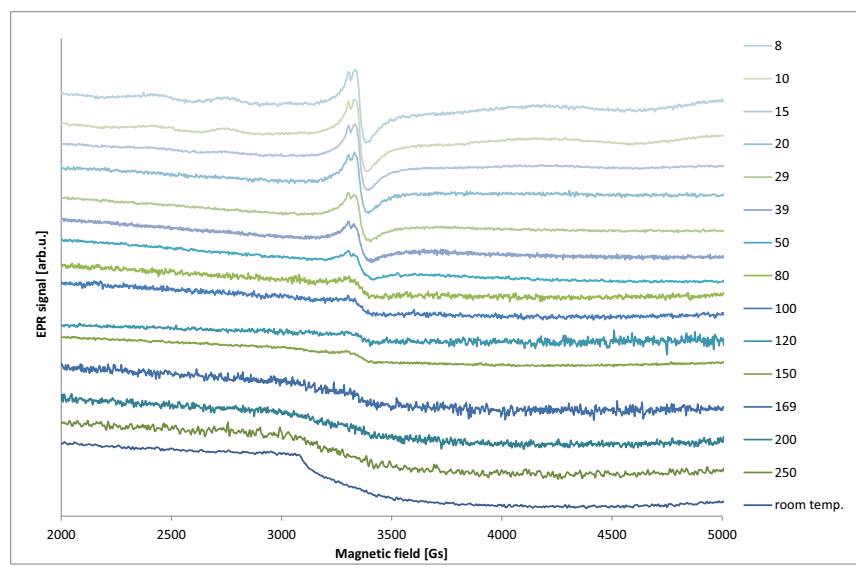

Fig. 3. The temperature dependence of EMR signal of the CNT.

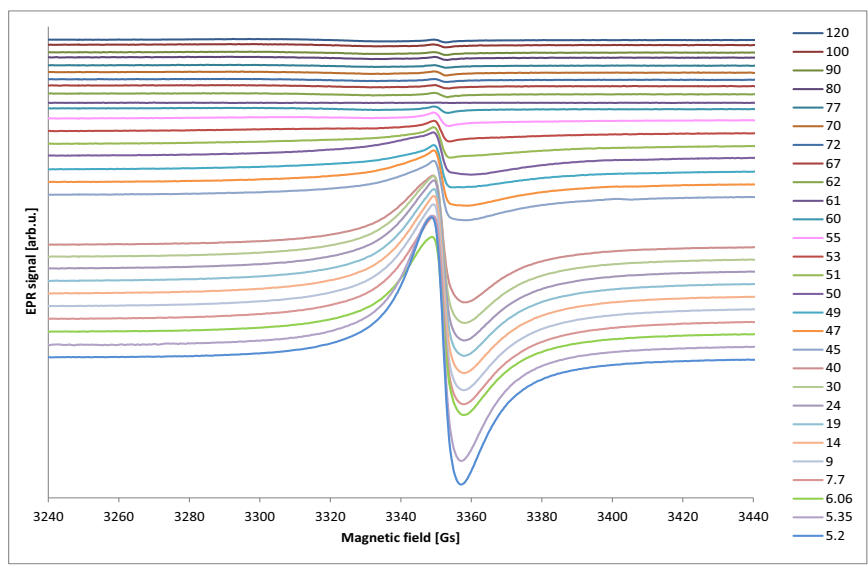

Fig. 4. The temperature dependence of EMR signal of the $\mathrm{CNH}$.

At low temperatures, the EMR spectrum of $\mathrm{CNH}$ changes its shape to Lorenzian with only slight asymmetry (adduct of the Dysonian component).

\section{Results and discussion}

The selected EMR spectra as a function of temperature for the CNT and CNH sample are shown in Fig. 3 and 4, respectively. The temperature dependencies of the peak to peak linewidth Bpp and the estimated integral intensity

$$
\mathrm{I}_{\text {int }}=\left(\mathrm{A} * \mathrm{~B}_{\mathrm{pp}}{ }^{2}\right)
$$

of the signal $(\mathrm{A}=\mathrm{a}+\mathrm{b})$ measured in the temperature interval $290-5.2 \mathrm{~K}$.

In Fig. 5 and 6 linewidth $\mathrm{B}_{\mathrm{pp}}$ for the $\mathrm{CNH}$ and $\mathrm{CNT}$ samples are presented respectively. 


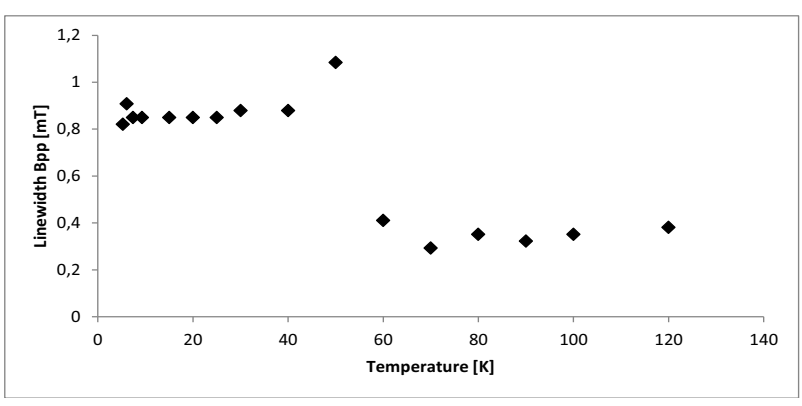

Fig. 5. The temperature dependence of linewidth $B_{p p}$ of the $\mathrm{CNH}$.



Fig. 6. The temperature dependence of line width $B_{p p}$ of the CNT.

In Fig. 7 and 8 integral intensity of EMR spectra obtained on eq. 1 for the $\mathrm{CNH}$ and CNT samples are presented respectively.



Fig. 7. The temperature dependence of integral intensity of the CNH.

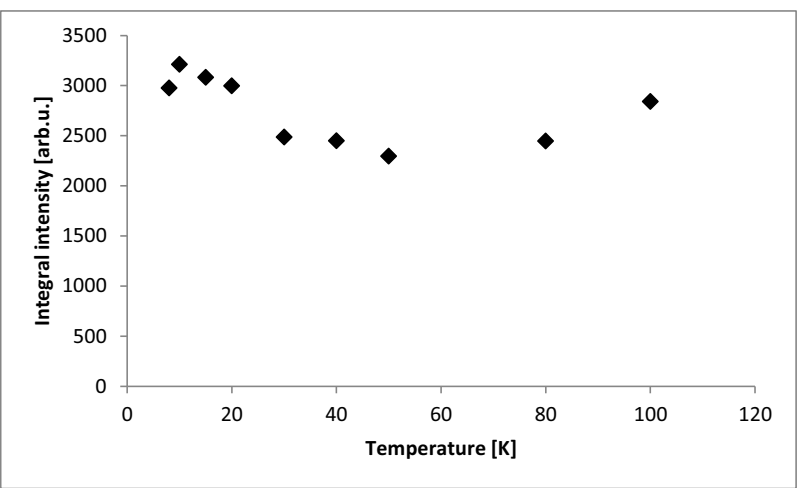

Fig. 8. The temperature dependence of integral intensity of the CNT.
The g-value was calculated using the magnetic field $\mathrm{B}_{0}$, which is obtained from the central position of the EMR spectral line. The g-value of $2.01-2.02$ is assigned to the interaction between the conducting electrons in the CNTs trapped at defects or magnetic ions site. The more the deviation from the freeelectron $\mathrm{g}$ value, the more is the localization due to defects [21]. In $\mathrm{CNH}$ and CNT samples, EMR lines are seen with the gvalue of $2.0027-1.999$ at $100 \mathrm{~K}, 2.002-2.000$ at $15 \mathrm{~K}$ respectively. Obtained value is very close to the free electron g-value (2.0023).

EMR is the perfect method to distinguish between the localized and conducting electrons and helps to determine the conducting properties of the carbon nanotubes and nanohorns. The conduction electrons can be considered as a Fermi gas. The alignment of magnetic moments with the external magnetic field is opposed not only by the thermal energy as for the isolated and independent electrons, but we also have to be sure that the Pauli principle is fulfilled. Therefore, the electron gas must obey to the Fermi-Dirac statistics [22]; consequently, the electrons that can be measured in a ESR experiment are those with energy near to the Fermi level. The others do not contribute. For this reason, the ESR spectra are usually less intense, regardless the number of electrons in the sample. The Fermi-Dirac statistic determines also the type of magnetism of these systems.

A solid that contains an ensemble of unpaired, noninteracting spins obeys to the Maxwell-Boltzmann statistic and its magnetic susceptivity obeys the Curie law $[22,23]$

$$
\chi=\frac{N v \mu^{2}}{3 k_{T} T} \mu_{0}=\frac{C}{T} \geq 0
$$

where $\mu_{0}=g^{2} \beta e 2 S(S+1)$ and $N_{V}$ is the number of magnetic species per unit volume. $C$ is the Curie constant and is characteristic of each paramagnetic substance through $\mu$. Usually, real solids do not obey exactly the Curie law; often the dependence of the magnetic susceptivity from the temperature has the following form

$$
\chi=\frac{C}{T-\theta}
$$

this is the Curie-Weiss law and the parameter $\theta$, the Weiss temperature, depends on the specific material and it is positive for materials that have a ferromagnetic behavior and negative for antiferromagnetic materials. The Curie-Weiss law comes as direct consequence of the dipolar magnetic interaction between the spin in the materials. Conductive systems have a magnetic suceptivity that is lower in module with respect to the systems with localized electrons and it doesn't depend on the temperature; in this case we have the Pauli paramagetism $[22,23]$.

A linear increase of $\chi-1(\mathrm{~T})$ at higher temperatures can be fitted to the Curie-Weiss law.

$$
(\chi(T)-\chi(0))^{-1}=\frac{T-\theta}{C}
$$

where $\mathrm{C}$ is the Curie constant, $\theta$ is the paramagnetic Curie temperature, and $\chi(0)$ is a temperature independent term to account for the diamagnetic host and any Pauli paramagnetism contribution. 




Fig. 9. Temperature dependence of the $1 /$ (integral intensity) of CNH sample

Figure 9 displays the temperature dependence of the quantity $(\chi(\mathrm{T})-\chi(0))-1$ on $\mathrm{CNH}$ sample. The line is a linear extrapolation illustrating the ferromagnetic (positive) Curie-Weiss temperature. Fitting yields the following values $\theta=46.6 \mathrm{~K}$ and $\mathrm{C}=384.6$ of $\mathrm{CNH}$. For CNT sample fitting yields the following values $\theta=7.7 \mathrm{~K}$ and $\mathrm{C}=1234.3$.

Therefore the EMR oscillations of CNT sample reflect jumplike displacements of impurities atom (i.e. oxygen) between walled carbon nanotubes. The amplitude of the oscillations depends on the number of electrons transferred from conduction band to localized state. Dysonian line characterized by two relaxation processes, relatively narrow Lorentzian line, characterized by one relaxation process. Such changes should modulate the conducting band as the result the conducting electrons give a broad EMR spectrum with fluctuating g-values.

The localized spin centers near defect states show Curie-like temperature dependence; by contrast, the EMR temperature dependence of conduction electron spins is close to that predicted by the Pauli magnetism. The comparison of EMR signal integral intensity at temperature dependence allows one to determine the relative contributions of both types of spin centres.

\section{CONCLUSION}

The asymmetric resonance lines with Dyson shape with an asymmetry parameter of the resonance line $\mathrm{a} / \mathrm{b}=1.5$ at $50 \mathrm{~K}$ were observed.

The g-value of multiwalled carbon nanotubes (CNT) and carbon nanohorns $(\mathrm{CNH})$ was calculated. In $\mathrm{CNH}$ and CNT sample EMR lines are seen with the g-value of $2.0027-1.999$ at $100 \mathrm{~K}, 2.002-2.000$ at $15 \mathrm{~K}$ respectively.

Analysis of the temperature dependences of the integral intensity of EMR spectra was carried out using the Curie-Weiss law and Curie temperature $\theta_{(\mathrm{CNT})}=46,6$ $\mathrm{K}$ and $\theta_{(\mathrm{CNT})}=8.6 \mathrm{~K}$ were obtained.

The localized spin centers near defect states show Curie-like temperature dependence; by contrast, the EMR temperature dependence of conduction electron spins is close to that predicted by the Pauli magnetism.
The comparison of EMR signal integral intensity at temperature dependence allows one to determine the relative contributions of both types of spin centres.

\section{References}

1. S. Iijima, Nature 56, 354 (1991)

2. M.S. Dresselhaus, G. Dresselhaus, P.C. Eklund, Science of Fullerenes and Carbon Nanotubes (Academic, New York, 1995)

3. M. Monthieoux, Carbon 40, 1809 (2002)

4. K. Esfarjani, A.A. Farajian, F. Ebrahimi, Y. Kawazoe, Eur. Phys. J. D 16, 353 (2001)

5. K. Tsukagoshi, I. Yagi, Y. Aoyagi, Appl. Phys. Lett. 85, 1021 (2004)

6. S. Hao, G. Zhuo, J. Wu, W. Duan, B.L. Gu, Phys. Rev. B 69, 113403 (2004)

7. A.C. Dillon, Nature 386, 377 (1997)

8. C. Liu, Y.Y. Fan, M. Liu, H.T. Cong, H.M. Cheng, M.S. Dresselhaus, Science 286, 1127 (1999)

9. W.A. Heer, A. Chatelain, D. Ugarte, Science 270, 1179 (1996)

10. S.J. Tans, A.R.M. Verschueren, C. Dekker, Nature 393, 49 (1998)

11. W.B. Choi, D.S. Chung, J.H. Kang, H.Y. Kim, Y.W. Jin, I.T. Han, Y.H. Lee, J.E. Jung, N.S. Lee, G.S. Park, J.M. Kim, Appl. Phys. Lett. 75, 3129 (1999)

12. S.R. Mishra, H.S. Rawat, S.C. Mehendale, K.C. Rustagi, A.K. Sood, R. Bandyopadhyay, A. Govindaraj, C.N.R. Rao, Chem. Phys. Lett. 317, 510 (2000)

13. O.V. Yazyev, Reports on Progress in Physics 73, 056501 (2010)

14. W. Kempiński, S. Łoś, M. Kempiński, D. Markowski, Beilstein J. Nanotechnol. 5, 1760 (2014)

15. W. Kempiński, M. Kempiński, D. Markowski, S. Łoś, Nukleonika 58, 3, 371 (2013)

16. M.A. Augustyniak-Jabłokow, Y.V. Yablokov, B. B. Andrzejewski, W. Kempiński, S. Łoś, K. Tadyszak, M.Y. Yablokov, V.A. Zhikharev. Phys Chem Minerals 37, 237 (2010)

17. V. Likodimos, S. Glenis, N. Guskos, C.L. Lin, Physical Review B 68, 045417 (2003)

18. S. Glenis, V. Likodimos, N. Guskos, C.L. Lin, Journal of Magnetism and Magnetic Materials 272276, 1660 (2004)

19. V. Likodimos, S. Glenis, N. Guskos, C.L. Lin, Physical Review B 76, 075420 (2007)

20. F.J Dyson., Physical Review. 98, 349 (1955)

21. D.K. Singh, P.K. Iyer, P.K. Giri, J. Nanosci. Nanotechnol. 9, 1 (2009)

22. N.W Ashcroft, N.D Mermin, Solid State Physics (Saunders College, Philadelphia, 1976)

23. A.F Orchard, Magnetochemistry (Oxford University Press, Oxford, 2003) 